Revisão dos registros da andorinha-do-rio Tachycineta albiventer (Aves: Hirundinidae) para a Região Metropolitana de São Paulo, sudeste do Brasil

Review of the records of the White-winged Swallow Tachycineta albiventer (Aves: Hirundinidae) for the São Paulo Metropolitan Region, southeastern Brazil

\author{
FÁBio Toledo das Dores ${ }^{1,6}$ \\ ROMEU GAMA ${ }^{2}$ \\ Marco Aurélio Galvão da Silva ${ }^{3}$ \\ PATRICK InÁCIO PINA ${ }^{4}$ \\ $\&$ FABIO SCHUNCK ${ }^{5}$ \\ 6. fabio_tol@msn.com
}

1. Centro de Triagem de Animais Silvestres, Parque Ecológico do Tietê - PET, São Paulo, SP, Brasil. 2. Parque Ecológico do
Tietê - PET, São Paulo, SP, Brasil. 3. Sociedade para a Conservação das Aves do Brasil - Save Brasil, São Paulo, SP, Brazil. 4.
Pesquisador independente. 5. Comitê Brasileiro de Registros Ornitológicos - CBRO (www.cbro.org.br).

A família Hirundinidae é representada por 19 gêneros e 86 espécies, que ocorrem em todas as partes do mundo, exceto em algumas regiões polares (WINKLER ET AL., 2021). A andorinha-do-rio (Tachycineta alviventer) é uma espécie endêmica da América do Sul e do Caribe, onde possui uma ocorrência ampla, sendo ausente apenas no Chile, Uruguai e no extremo sul do continente (SEDGWICK, 2021). Com cerca de $13,5 \mathrm{~cm}$ e 20,5 g, possui penas brancas bem evidentes nas asas e uropígio, que contrastam com a cor verde azulada do corpo, sendo encontrada em diferentes ambientes aquáticos como lagoas, estuários, rios, canais e (c) (i) Copyright by the Author. This is an open-access article distributed under the terms of the Creak 
manguezais, tanto do interior como do litoral. Se alimenta de pequenos insetos que captura em voo rente ao espelho d'água, sendo observadas sozinhas, em pares e mais raramente em pequenos grupos (BELTON, 1985; SigRIST, 2006; SEDGWICK, 2021).

A andorinha-do-rio (Tachycineta alviventer) ocorre em todas as regiões do Brasil, sendo comum na Amazônia e localmente migratória no Rio Grande do Sul, onde desaparece no inverno, em busca de áreas mais propícias para alimentação ao norte (BeLton, 1985; Sick, 1997). No entanto, sua possível migração no sul do país ainda é pouco estudada, não sendo mencionada na revisão feita por SoMENZARI ET AL. (2018). No estado de São Paulo é residente, e apresenta uma ocorrência voltada principalmente aos grandes rios do interior, como Paraná e Tietê, mas também está presente no Vale do rio Ribeira de Iguape e litoral sul, onde foi registrada em 1898 por Ernst Richard Krone (1861-1917) (PINTO, 1944; WILLIS \& ONIKI, 2003). Nas demais áreas costeiras do estado, a ocorrência de $T$. alviventer é variada, sendo considerada incomum no litoral norte por Simpson et AL. (2012), ausente em Bertioga (e.g., CAVARZERE ET AL., 2014; BoKermann \& Pivelli, 2019; BoKermann et AL., 2021), registrada apenas recentemente em Cubatão, que é uma das áreas mais estudadas ornitologicamente do litoral paulista (Silva E SiLVA \& Olmos, 2020), com status indefinido para Mongaguá e Itanhaém (Lima, 2010) e ausente na região da Juréia-Itatins (DEVELEY, 2004).

A andorinha-do-rio também continua sem registros para a Região Metropolitana de São Paulo (RMSP), no leste do estado, onde temos a maior área urbana da América do Sul (Willis \& ONIKI, 2003; IBGE, 2018; Figueiredo, 2020; São Paulo, 2018). Com base neste cenário e com o objetivo de entender a ocorrência de Tachycineta albiventer na RMSP, resgatamos, organizamos e avaliamos dados disponíveis em diferentes fontes, incluindo a descrição do primeiro registro de campo documentado para o município de São Paulo.

\section{Dados secundários}

\section{MATERIAL E MÉTODOS}

Os dados secundários de ocorrência foram obtidos por meio de buscas em artigos científicos, livros, literatura cinza e coleções ornitológicas disponíveis no banco de dados GBIF (Global Biodiversity Information Facility - https://www.gbif.org). Foram utilizadas combinações de palavras chave como Tachycineta alviventer, andorinha-do-rio, White-winged 
http://dx.doi.org/10.5380/abp.v50i1-4.82452

Swallow e São Paulo. Também foram consultadas as principais plataformas ornitológicas online com dados do Brasil e do estado de São Paulo, como WikiAves (https://wikiaves.com.br), eBird (https://ebird.org), Xeno-Canto (https://xeno-canto.org) e Táxeus (https://taxeus.com.br), até 27 de junho de 2021. Os registros sem localidades específicas foram atribuídos ao ponto central do respectivo município. Os registros com documentação (foto e/ou gravação) foram checados quanto a identificação correta da espécie.

\section{Dados de campo}

Os dados de campo foram produzidos no Parque Ecológico do Tietê PET, núcleo Engenheiro Goulart (2329'23.15”S, 46³1'10.90”W 740 m a.n.m.), uma unidade de conservação estadual criada em 1976 e inaugurada em 1982, com 1.250 ha, localizada na região leste do município de São Paulo, na divisa com o município de Guarulhos (DEVECCHI, 2010) (Fig. 1). O parque protege um trecho com cerca de $8,5 \mathrm{~km}$ do rio Tietê e possui alguns lagos artificiais, áreas alagadas, ambientes abertos e uma variedade de ambientes florestais em diferentes estágios sucessionais, incluindo vegetação nativa (Mata Atlântica) e exótica (e.g., pinheiros,

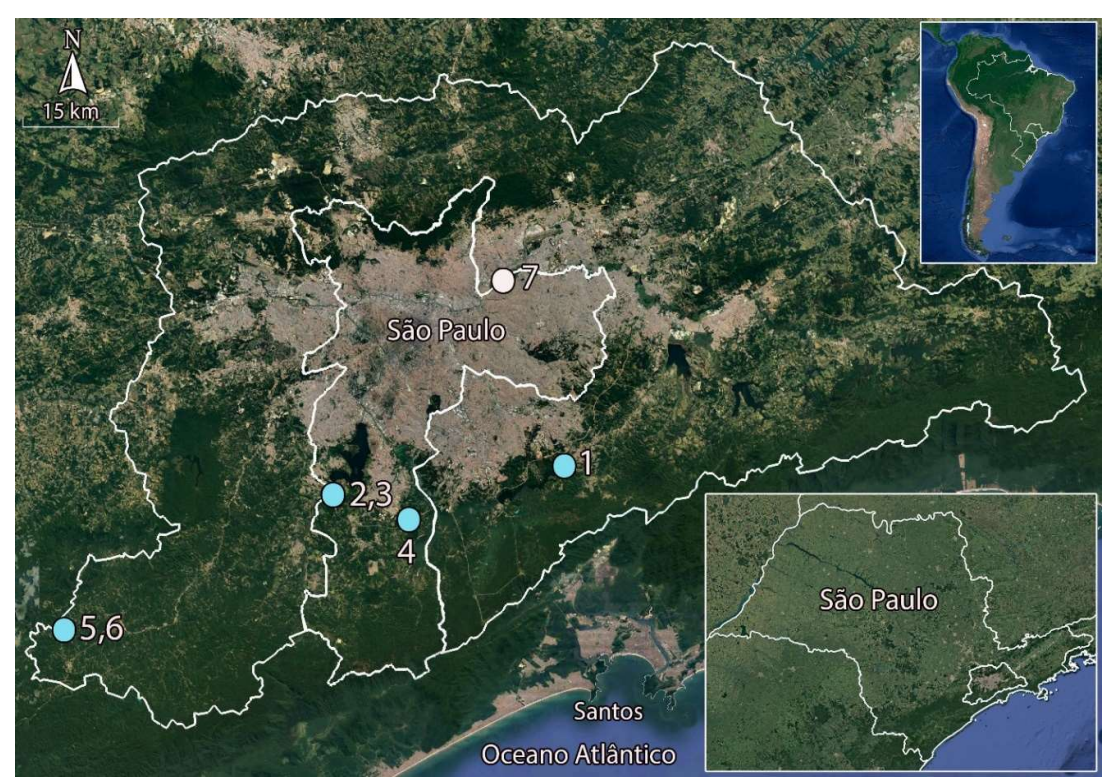

Figura 1. Mapa da Região Metropolitana de São Paulo, com os registros históricos (azuis) e atuais (branco) de Tachycineta albiventer. Os números são referentes aos dados da Tabela 1, sendo que o número 7 é o Parque Ecológico do Tietê PET. 
eucaliptos), que proporcionam a ocorrência de uma rica avifauna, com cerca de 274 espécies já detectadas, incluindo táxons residentes e migratórios (DORES ET AL., 2020).

\section{RESULTADOS}

Foram obtidos seis registros de Tachycineta albiventer para a Região Metropolitana de São Paulo, feitos entre 1999 e 2015, sendo três no inverno, um no verão e dois em períodos não determinados (sem datas específicas), sendo todos sem documentação. Os respectivos registros foram obtidos na literatura cinza, em relatório de impacto ambiental (01 São Paulo, 2005), relatório de monitoramento de aves (02 - Ecologic, 2013) e na base de dados online do Centro de Estudos Ornitológicos CEO (03 - CEO, 2014), referente as excursões de observação de aves feitas pelos associados dessa ONG no estado de São Paulo (Fig. 1; Tabela $1)$.

Tabela 1. Registros históricos e atuais de Tachycineta albiventer na Região Metropolitana de São Paulo. Os registros 2 e 3 foram organizados aleatoriamente, pois não apresentam datas específicas. As localidades 5 e 6 possuem a mesma coordenada geográfica devido à proximidade.

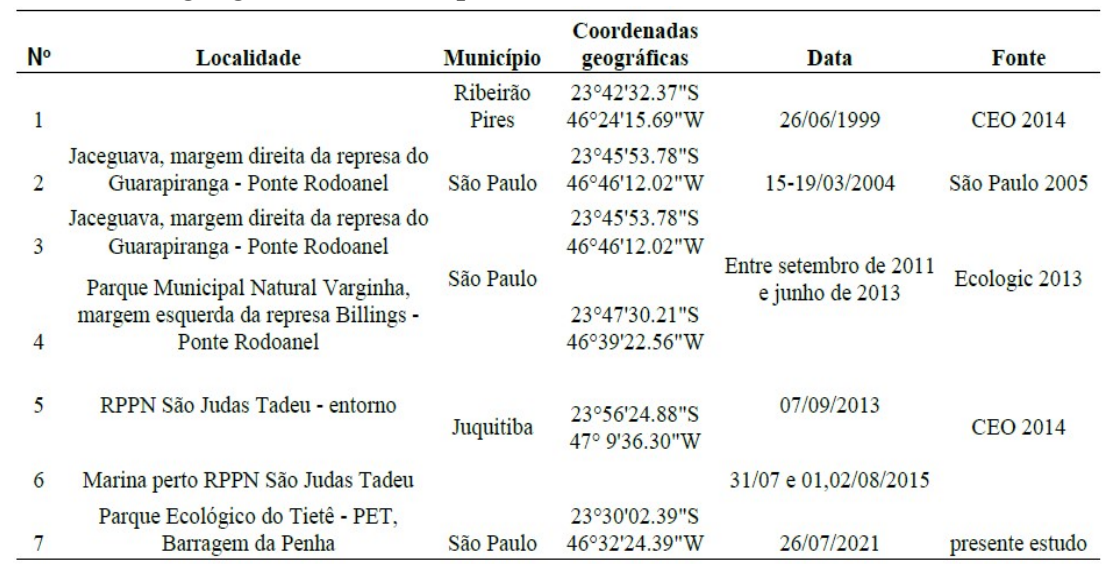

No dia 26 de junho de 2021, por volta das 11:15 da manhã, os autores observaram um indivíduo adulto de Tachycineta albiventer sobrevoando o rio Tietê próximo da Barragem da Penha, no Parque Ecológico do Tietê - PET (2330'3.17"S, 46³2'25.83"W 740 m) (Fig. 2A). O indivíduo estava se alimentando em voos sobre o espelho d'água e bancos de sedimento existentes no leito do rio, utilizando um semáforo náutico como ponto de pouso (Fig. 2B e C e ML350481681). O indivíduo foi observado durante 
http://dx.doi.org/10.5380/abp.v50i1-4.82452

cerca de 30 minutos, forrageando juntamente com um grupo de andorinhapequena-de-casa (Pygochelidon cyanoleuca), depois não foi mais visto. Estas observações foram feitas durante uma saída de campo para observação de aves e possíveis detecções de espécies visitantes de inverno na cidade de São Paulo, sendo detectadas 81 espécies (https://ebird.org/ checklist/S90808069). Na semana seguinte ao primeiro registro, nos dias

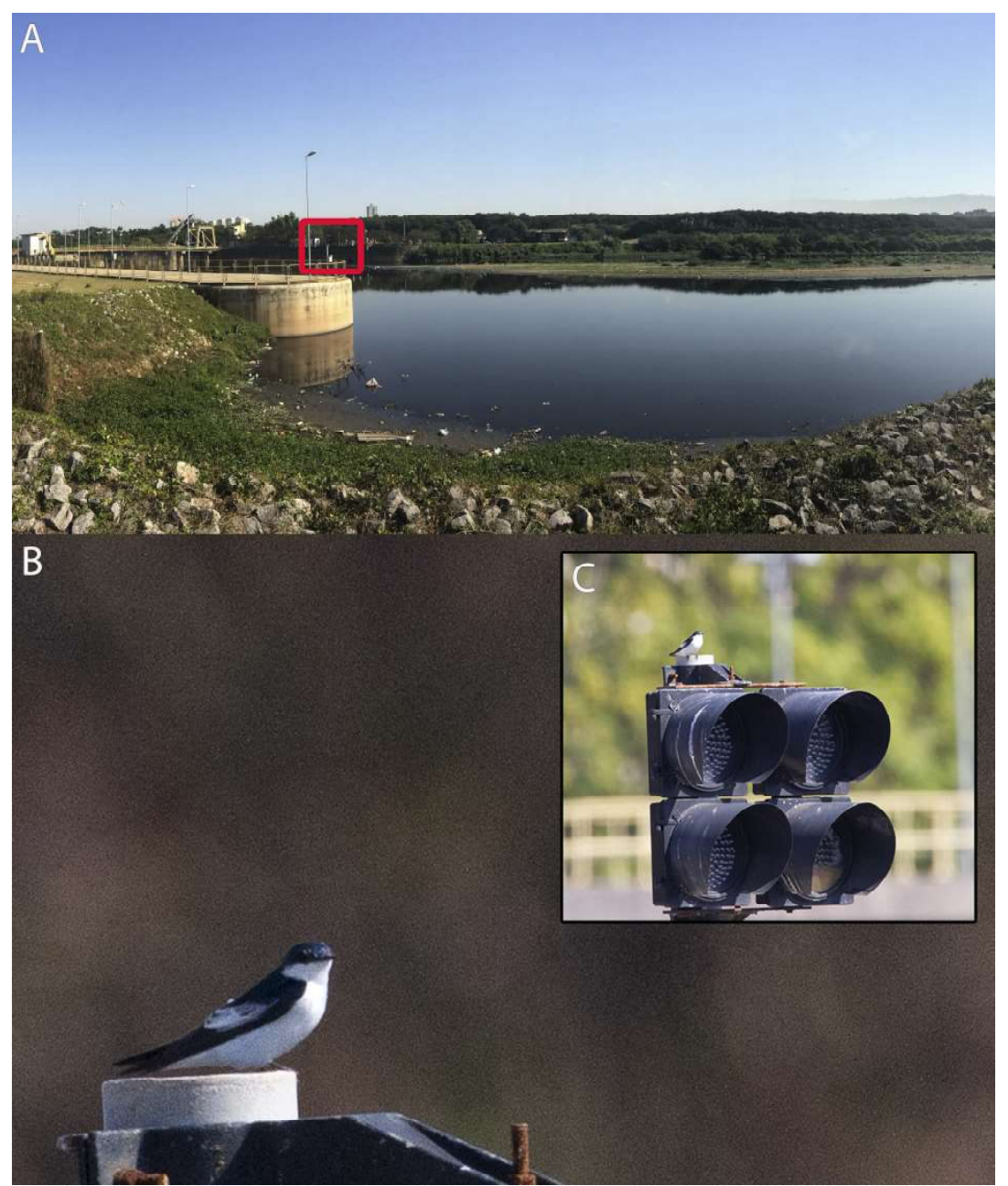

Figura 2. A. Região da Barragem da Penha, no rio Tietê, localizada no limite do Parque Ecológico do Tietê. O quadrado vermelho indica o local do semáforo náutico, onde o indivíduo de Tachycineta alviventer foi registrado; B. Indivíduo de T. alviventer fotografado e C. detalhe domesmo indivíduo fotografado. Fotos: Fabio Schunck. 
28 e 30 de junho e 4 de julho, o autor FTD fez novas visitas na Barragem da Penha, mas o indivíduo de T. albiventer não foi detectado.

\section{DISCUSSÃO}

Os registros históricos de Tachycineta albiventer disponíveis para a Região Metropolitana de São Paulo (6), mostram se tratar de uma espécie incomum, com ocorrência voltada a reservatórios hídricos artificiais (Billings, Guarapiranga e do rio Juquiá) e detectada a pouco mais de 20 anos. Isso pode ser concluído com base nas ocorrências de outras andorinhas comuns que vivem no mesmo tipo de ambiente (e.g., andorinha-serradora Stelgidopteryx ruficollis e andorinha-de-sobre-branco Tachycineta leucorrhoa) e que vem sendo registradas na região ao longo dos últimos 200 anos (Pelzeln, 1871; Ihering, 1898; Figueiredo, 2020). A ausência de dados publicados em periódicos científicos com revisão por pares, fez com que a presença dessa espécie passasse despercebida na RMSP por muitos anos, prejudicando uma análise mais efetiva sobre seu status de ocorrência nessa região do estado de São Paulo, ficando de fora de listas e revisões regionais que são utilizadas em ações de conservação, como do município de São Paulo (SCHUNCK, 2008; Figueiredo, 2020).

O registro de Tachycineta albiventer feito no Parque Ecológico do Tietê, é o sétimo para a RMSP, o quarto para o município de São Paulo, sendo o primeiro com documentação e o primeiro para um rio, pois os anteriores foram nos reservatórios Guarapiranga e Billings. Entre os sete registros disponíveis para a RMSP, três foram feitos em unidades de conservação, como o Parque Municipal Natural Varginha (4), RPPN São Judas Tadeu (5) e Parque Ecológico do Tietê, mostrando a importância destas UCs na proteção dos últimos remanescentes de várzea da RMSP, que mesmo com ambientes degradados e poluídos, ainda possuem condições para abrigar espécies incomuns, migratórias e residentes.

O baixo número de registros dessa espécie para a RMSP (7) nos faz pensar em alguns pontos principais sobre sua ocorrência, como detectabilidade, migração, clima e habitat. Podemos descartar a possibilidade de algum tipo de problema de detecção de campo, pois devido a sua plumagem típica e distinta das demais andorinhas, não passaria despercebida por tantos anos, pois a RMSP é historicamente a região do estado de São Paulo com a maior concentração de pesquisadores, observadores e fotógrafos de aves (SCHUNCK, 2008; EBIRD, 2021; WIKIAvES, 2021). Os dados disponíveis para a região leste de São Paulo, mostram uma presença em todas as estações do ano (EBIRD, 2021; WIKIAves, 
http://dx.doi.org/10.5380/abp.v50i1-4.82452

2021), descartando uma ocorrência migratória ampla e bem conhecida, como o tesourinha Tyrannus savana, mas o fato do maior número de registros da RMSP ter sido feito no inverno, incluindo no PET, não descartamos a possibilidade de existir algum tipo de movimentação regional no período mais frio e seco do ano, como já observado com outras espécies de aves no município de São Paulo (SCHUnCK \& Alves, 2020; SCHUNCK ET AL., 2020). Estudos de captura, marcação e recaptura, ou mesmo que utilizem geolocalizadores e gps, poderão elucidar melhor essa questão da existência ou não de movimentações regionais realizadas pelas andorinhas-do-rio.

As questões climáticas e geográficas também parecem não ser possíveis explicações para justificar algum tipo de ocorrência dessa espécie no estado de São Paulo. Quando separamos interior e litoral, temos um número maior de registros no interior, que é uma região mais quente, onde inclusive a espécie se reproduz, porém, a área geográfica é maior, com grandes rios e mais ambientes, facilitando sua ocorrência. No caso do litoral, mesmo sendo uma região mais fria e com um número menor de registros, temos uma área mais reduzida (incluindo o limite com o mar), com menos ambientes disponíveis. Mesmo assim, temos dois cenários distintos, a região sul, entre Cananéia e Iguape, incluindo o Vale do rio Ribeira de Iguape, com uma certa frequência de registros históricos e atuais ao longo do ano, e a região central e norte, com registros ocasionais e esporádicos (Willis \& ONIKI, 2003; CAVARZERE ET AL., 2014; BOKERMANN \& Pivelli, 2019; Silva E Silva \& Olmos, 2020; Bokerman et Al., 2021; EBIRD, 2021; WiKIAVES, 2021). Uma diferença entre estas duas regiões é o tamanho da área de planície existente, que no sul, é formada pela região litorânea e o Vale do Ribeira, se estendendo da costa até a Serra de Paranapiacaba (cerca de $70 \mathrm{~km}$ ), com a presença do rio Ribeira de Iguape (de porte médio) e seus tributários, formando uma ampla variedade de ambientes. Já entre Peruíbe e Ubatuba, existe uma diminuição da área da planície litorânea e seus respectivos ambientes entre o sul (cerca de $15 \mathrm{~km}$ ) e o norte (com vários trechos da costa sem planície), com menos corpos hídricos de porte médio, coincidindo com a diminuição dos registros de T. albiventer (Willis \& ONIKI, 2003; EBIRD, 2021; WiKIAVES, 2021). Estudos que considerem variações climáticas, de habitat e de paisagem também poderão ser importantes para ajudar a entender a distribuição geográfica da andorinha-do-rio.

Em relação ao habitat, a RMSP sempre apresentou uma diversidade de ambientes húmidos, incluindo rios de pequeno e médio porte, além de 
reservatórios artificiais com mais de 100 anos, com ocorrências históricas e atuais de Stelgidopteryx ruficollis e T. leucorrhoa, que apresentam características biológicas e ecológicas semelhantes a T. albiventer (SICK, 1997; FrançA, 2000; Figueiredo, 2020). Mesmo a RMSP tendo se tornado a maior área urbana da América do Sul, com cerca de 20 milhões de habitantes (IBGE, 2018), com redução de habitat natural e aumento da poluição de corpos hídricos (FrANÇA, 2000), estes não seriam impedimentos para sua ocorrência, pois essa andorinha vem sendo detectada em áreas urbanas e rios poluídos do interior do estado, como o próprio rio Tietê, no município de Salto, onde temos 34 registros disponíveis (WIKIAves, 2021). No entanto, a maior parte da diversidade de ambientes utilizados pela espécie está no interior do estado e no Vale do rio Ribeira de Iguape, regiões onde temos a presença de rios de porte médio e grande, como Grande, Tietê, Paraná, Paranapanema e Ribeira de Iguape. Mesmo essa espécie não sendo exclusivamente dependente dos rios de médio e grande porte, como a mexeriqueira (Vanellus cayanus), a falta deste tipo de corpo hídrico na RMSP, pode ser um dos principais motivos pela sua ausência histórica e ocorrência incomum atual, característica que inclusive a diferencia de Stelgidopteryx ruficollis e T. leucorrhoa.

Os dados históricos e atuais de Tachycineta albiventer disponíveis para a RMSP, revelam um cenário curioso e pouco conhecido sobre a ocorrência incomum dessa espécie na maior área urbana da América do Sul. O resgate, a avaliação técnica e a publicação de dados históricos deve ser um processo contínuo, pois ainda existem muitas informações ornitológicas dispersas em fontes pouco acessíveis, que quando comparadas com dados atuais, incluindo plataformas ornitológicas online, podem revelar padrões desconhecidos e ampliar o conhecimento sobre a ocorrência de muitas espécies de aves do estado de São Paulo. No caso das espécies que utilizam ambientes úmidos, como a andorinha-do-rio, a necessidade de revisão dos dados e detecção de áreas de ocorrência passa a ser prioridade, pois são ambientes que estão entre os mais ameaçados do mundo e precisam urgentemente de proteção legal sob a forma de unidades de conservação (SHENGIIE ET AL., 2017).

Sugerimos algumas ações que possam beneficiar a obtenção do conhecimento sobre a andorinha-do-rio na Região Metropolitana de São Paulo e demais áreas de ocorrência, como continuar resgatando e publicando registros da literatura cinza e outras fontes restritas, incluindo dados de terceiros, colocando-os a luz de novas interpretações; realizar censos populacionais em diferentes regiões, como no litoral sul de São 
Acta Biol. Par., Curitiba, 50 (1-4): 103-115. 2021

http://dx.doi.org/10.5380/abp.v50i1-4.82452

Paulo (incluindo o Vale do rio Ribeira de Iguape), para tentar entender se existe algum tipo de movimentação sazonal em alguma época do ano; investir em estudos que considerem variáveis climáticas e ambientais para entender a distribuição geográfica dessa espécie e possíveis preferências de habitat, assim como trabalhos de marcação (captura e recaptura) e uso de tecnologias como geolocalizadores e gps para investigar possíveis movimentações regionais; fazer uma análise ampla sobre a ocorrência dessa espécie no Brasil, testando as hipóteses disponíveis sobre sua possível migração no sul do país e proteger os últimos remanescentes de várzea da Região Metropolitana de São Paulo, incentivando a criação de novas unidades de conservação ao longo dos rios existentes, como o Tietê.

\section{AGRADECIMENTOS}

Aos funcionários do PET pelo apoio logístico de campo; ao Luiz Fernando Figueiredo do CEO, pelos esclarecimentos sobre as informações disponíveis na plataforma de dados dessa instituição.

The author declares no conflicts of interest.

\section{SUMÁRIO}

A família Hirundinidae apresenta uma ampla distribuição ao redor do mundo, com táxons exclusivos de algumas regiões, como a andorinhado-rio (Tachycineta alviventer), endêmica da América do Sul e Caribe. Essa espécie ocorre em todo o Brasil, incluindo o estado de São Paulo, mas sua presença na Região Metropolitana de São Paulo (RMSP), onde se encontra a maior área urbana da América do Sul, ainda não foi detectada. Com base neste cenário, apresentamos uma revisão dos registros históricos e a primeira detecção de campo documentada dessa espécie para a RMSP. Foram consultados dados da literatura, museus de história natural e plataformas ornitológicas online, sendo possível obter apenas seis registros históricos, produzidos entre 1999 e 2015. O registro documentado de campo foi feito em junho de 2021, no Parque Ecológico do Tietê, na área urbana da cidade de São Paulo. O baixo número de registros de $T$. albiventer para a RMSP (7) mostram se tratar de uma espécie incomum, que vem utilizando reservatórios hídricos artificiais ocasionalmente nos últimos 22 anos. Essa presença ocasional pode estar relacionada a ausência de rios de médio e grande porte na RMSP e no seu entorno direto, sendo um comportamento diferente de outras espécies de andorinhas que ocupam o mesmo tipo de habitat. Essa questão merece 
uma investigação mais ampla e os ambientes úmidos da Região Metropolitana de São Paulo precisam de uma proteção legal emergencial, pois apresentam uma alta riqueza de espécies de aves.

PalaVRas-Chave: Conservação, ambientes úmidos, reservatórios hídricos, migração,

\section{SUMMARY}

The Hirundinidae family has a wide distribution around the world, with taxa that are exclusive to some regions, such as the White-winged Swallow (Tachycineta alviventer), endemic to South America and the Caribbean. This species occurs throughout Brazil, including the state of São Paulo, but its presence in the São Paulo Metropolitan Region (SPMR), where the largest urban area in South America is located, has not yet been detected. Based on this scenario, we present a review of the historical records and the first documented field detection of this species for the SPMR. Data from literature, natural history museums and online ornithological platforms were consulted. We accessed six records, produced from 1999 and 2015. The documented field record was made in June 2021, at Tietê Ecological Park, in the urban area of the city of São Paulo. The low number of records of T. albiventer for the SPRM (7) show that it is an uncommon species, which has been an occasional water reservoirs user in the last 22 years. This occasional presence may be related to the absence of medium and large rivers in the SPRM and its direct surroundings, which is a different requirement compared to other species of swallows that occur the same type of habitat. The lack of occurrences for White-winged Swallow suggests that we need more research in the area, along with an increase in the protected areas in the surroundings, as they present a high bird species diversity.

\section{BIBLIOGRAFIA}

Belton, W. 1985. Birds of Rio Grande do Sul, Brazil. Part 2. Bulletim American Museum of Natural History, 180:1-241.

BoKermanN, M. \& S.R.P. Pivelli. 2019. Avifauna de Bertioga - Inventários do Clube de Observação de Aves de Bertioga. UNISANTA Bioscience, 8 (3): 218-249.

Bokermann, M.; S. Pivelli; O. Freitas-Júnior. \& F. Schunck. 2021. Aves de Itaguaré: a importância das restingas e estuários de Bertioga para a conservação das aves do estado de São Paulo, sudeste do Brasil. Atualidades Ornitológicas, 218: 43-61.

Cavarzere, V.; T.V.V. Costa; G.A. Benedicto; L. Moreira-Lima \& L.F. SilveIRA. 2014. Continued bird surveys in southeastern coastal Brazilian Atlantic forests and the importance of conserving elevational gradients. 
http://dx.doi.org/10.5380/abp.v50i1-4.82452

Revista Brasileira de Ornitologia, 22 (4): 381-407.

Centro de Estudos Ornitológicos - CEO 2014. Registros ornitológicos em localidades do estado de São Paulo. Versão 20/12/2014. Disponível em: $<$ http://www.ceo.org.br>. Acesso em: 27 jun. 2021.

Devecchi, A. 2010. Os parques lineares e a oportunidade que São Paulo não pode perder. In: São PaUlo (ORG.) Guias dos parques municipais de São Paulo. vol. 2, pp. 157-160.

Develey, P.F. 2004. As aves da Estação Ecológica Juréia-Itatins. IN: Marques, O. A. V. \& W. Duleba. (eds). Estação Ecológica JuréiaItatins. Ambiente fisico, flora e fauna. Ribeirão Preto: Holos. pp. 278-295.

Dores, F. T.; L.S. Fitorra; V.S. Pedro; L.G.R. Longo; L.D.C. Pivotto; H.R. Furuya; B.S.S. Petri \& L. Milanelo. 2020. Avifauna do Parque Ecológico do Tietê núcleo Engenheiro Goulart, São Paulo, SP, Brasil. Revista Ciência, Tecnologia \& Ambiente, 10: e10155.

EBIRD. 2021. eBird: An online database of bird distribution and abundance [web application]. eBird, Cornell Lab of Ornithology, Ithaca, New York. Disponível em: <http://www.ebird.org>. Acesso em: 03 jan. 2021.

Ecologic - Centro de Avaliações e Perícias Ambientais. 2013. Monitoramento de aves do trecho sul do Rodoanel Mário Covas, SP. Relatório Consolidado.

Figueiredo, L.F.A. 2020. Lista das aVes do MunicíPio de São Paulo. Versão 07/07/2020. Disponível EM: <HTTP://CEO.ORG.BR/LISTAS_DE_AVES/ LISTA_MUNICIPIO_SP.XLS >.ACESSO EM: 27 NOV. 2020.

FrançA, E. 2000. Guarapiranga: recuperação urbana e ambiental do município de São Paulo. São Paulo: M. Carrilho Arquitetos. 256.

IBGE - Instituto Brasileiro de Geografia e Estatística. 2018. Instituto Brasileiro de Geografia e Estatística: Estimativas da população residente para os municipios e para as unidades da federação com data de referência em 10 de julho de 2018. Ministério do Planejamento, Desenvolvimento e Gestão. Distrito Federal, Brasil.

Ihering, H. 1898. As aves do Estado de São Paulo. Revista do Museu Paulista, 3: 113-476.

LimA, B. 2010. A avifauna das florestas de restinga de Itanhaém/ Mongaguá, estado de São Paulo, Brasil. Atualidades Ornitológicas, 153: 50-54.

Pelzeln, A. 1871. Zur Ornithologie Brasiliens. Resultate von Johann Natterers Reisen in den jahren 1817 Bis 1835. Vien: Druck und Verlag von A. Pichler's Witwe \& Sohn. 
Pinto, O.M.O. 1944. Catálogo das aves do Brasil e lista dos exemplares existentes na coleção do Departamento de Zoologia: 2 a Parte. São Paulo: Secretaria da Agricultura/Departamento de Zoologia, 1944.

São Paulo (estado). 2005. Complementação do Estudo de Impacto Ambiental das Obras do Trecho Sul Modificado do Rodoanel Mário Covas. Atendimento ao Item $2 d$ do Termo de Referência do parecer Técnico IBAMA No 025/2005. Estudos da Fauna. Relatório Final. Fundação Escola de Sociologia e Política de São PauloFESPSP. Dersa. Secretaria dos Transportes. Governo do estado de São Paulo.

São Paulo (CIDAde). 2018A. Inventário da Fauna do Município de São Paulo. Divisão de Fauna Silvestre/SVMA/PMSP. 223p.

SEDGwick, C.W. 2021. White-winged Swallow (Tachycineta albiventer), version 1.0. In: Schulenberg, T.S. (ed.). Birds of the World. Cornell Lab of Ornithology, Ithaca, NY, USA. Disponível em: https:/doi.org/ 10.2173/bow.whwswa1.01. Acesso em: 27 jun. 2021.

Schunck, F. 2008. As aves do município de São Paulo: conhecimento histórico, diversidade e conservação. p. 270- 313. In: Malagoli L.R.; F.B. Bajesteiro \& M. Whately (eds.). Além do concreto: contribuições para a proteção da biodiversidade paulistana. São Paulo: Instituto Sócio Ambiental - ISA. 359p.

Schunck, F. \& A.R. Alves. 2020. Jacques Cousteau: a importância de um parque urbano para a conservação das aves do município de São Paulo, sudeste do Brasil. Atualidades Ornitológicas, 215: 47-66.

Schunck, F.; F. D’Addezio; C. Nucitelli \& P. Mix. 2020. Aves da Península do Castelo: a importância da coleta de dados de longo prazo em escala geográfica local no município de São Paulo, SP. Atualidades Ornitológicas, 214: 51-65.

Shengile, H.; N. Zhenquo; C. Yanfen; L. Lifeng \& Z. Haiying. 2017. Global wetlands: Potencial distribution, wetland loss, and status. Science of the Total Environment, 586: 319-327.

Sick, H. 1997. Ornitologia Brasileira. Rio de Janeiro: Nova Fronteira. 912p.

Sigrist, T. 2006. Aves do Brasil, uma visão artística. Vinhedo: Avisbrasilis. 672p.

Silva e Silva, R. \& F. Olmos. 2020. Novos registros para a avifauna dos manguezais de Santos e Cubatão (SP), sudeste do Brasil. Atualidades Ornitológicas, 213: 45-77.

Simpson, R.; V. CaVArzere \& E. Simpson. 2012. List of documented bird species from the municipality of Ubatuba, state of São Paulo, Brazil. 
Acta Biol. Par., Curitiba, 50 (1-4): 103-115. 2021

http://dx.doi.org/10.5380/abp.v50i1-4.82452

Papéis Avulsos de Zoologia, 52: 233-254.

Somenzari, M.; P. Amaral; V. Cueto; A. Guaraldo; A. Jahn; D. Lima; P. Lima; C. Lugarini; C.G. Machado; J, Martinez; J.L.X. Nascimento; F.F. Pacheco; D. Paludo; N. Prestes; P. Serafini; L.F. Silveira; A.E. SouzA; N.A. SouzA; M.A. SouzA; W. Telino-JúnIor \& B.M. WhitneY. 2018. An overview of migratory birds in Brazil. Papéis Avulsos de Zoologia, 58: 1-66.

WikiAves. 2021. WikiAves - A enciclopédia das aves do Brasil. Juiz de Fora (MG). Disponível em: <http://www.wikiaves.com.br>. Acesso em: 05 Jul. 2021.

Willis, E.O. \& Y. Oniki. 2003. Aves do Estado de São Paulo. Rio Claro: Ed. Divisa. 398p.

Winkler, D.W.; S.M. Billerman \& I.J. Lovette. 2021. Swallows (Hirundinidae), version 1.0. In: BILLERMAN, S.M.; B.K. KeEney; P.G. Rodewald \& T.S. Schulenberg (eds.) Birds of the World. Cornell Lab of Ornithology, Ithaca, NY, USA. https://doi.org/10.2173/ bow.hirund2.01. Acesso em: 27 jun.2021.

Received 15 August 2021

Accepted 20 October 2021

Available Online 22 October 2021

Edited by Rodrigo B. Gonçalves 
